\title{
The Viability and Performance of P300 Responses using Low Fidelity Equipment
}

\author{
Patrick Schembri, Richard Anthony, Mariusz Pelc \\ Department of Computer and Information Systems, University of Greenwich \\ Greenwich London, UK \\ P.Schembri@greenwich.ac.uk, R.J.Anthony@greenwich.ac.uk, M.Pelc@greenwich.ac.uk
}

\begin{abstract}
In this paper we investigate the viability, practicability and efficacy of eliciting P300 responses based on the P300 speller $\mathrm{BCI}$ paradigm (oddball) and the xDAWN algorithm using five healthy subjects; while using a non-invasive Brain Computer Interface (BCI) based on low fidelity electroencephalographic (EEG) equipment. In the past decade there was a proliferation of cheap EEG equipment, including user-made equipment, which exposed an evident necessity to validate the equipment's suitability. Moreover a number of researchers and end users are currently using off-the-shelf equipment as a "black box" approach without any qualitative testing. Part of our contribution will be to create awareness of what type of hardware components are being utilized in our low fidelity equipment, vis-à-vis the results achieved. Our main contribution is to assess the functionality and reliability of our low cost equipment in its ability to detect the P300 component in a consistent, reliable and effective manner as a basis for future studies. This work forms part of a wider project where we plan to introduce a number of distractions and assessing the ways and extents to which different degrees of distractions affect the detection success achievable of the P300 component while using our low cost equipment. Our results demonstrate the applicability of using this off-the-shelf equipment as a means to successfully and effectively detect P300 responses.
\end{abstract}

Keywords: Brain Computer Interface (BCI), Electroencephalography (EEG), Event-related potential (ERP), P300 Speller.

\section{Introduction}

In this paper we investigate the ability, practicability and efficacy of eliciting P300 responses based on the P300 speller BCI paradigm (oddball) and the xDAWN algorithm with five healthy subjects, while using low fidelity equipment. Our research makes use of a non-invasive Brain Computer Interface (BCI) on the basis of Electroencephalography (EEG). The work presented here is part of a larger EEG based project and in continuation of our previous paper [1] and aimed as a basis for future studies where we plan to introduce a number of distractions and assessing the ways and extents to which different degrees of distractions affect the detection success achievability of the P300 component while using our aforementioned equipment.

In this paper we report a study where five healthy subjects used Farwell \& Donchin P300 speller paradigm [2]; where we based the methodology on the xDAWN algorithm [3]; to communicate nine alphanumeric characters in lab conditions, while using specific low fidelity equipment. Our aim is to assess the functionality and reliability of our low cost equipment in its ability to detect the P300 component in a consistent, reliable and effective manner as a basis for future studies.

\section{Experimental Methodology}

The work presented in this paper will make use of non-invasive BCI on the basis of EEG and will be utilizing specific low-fidelity equipment in lab conditions. The objective is to perform quantitative and qualitative analysis on the elicited P300 responses and establish to which extent these can be detected within our specific low cost equipment. In our experiments we have opted to use Farwell and Donchin P300 speller BCI paradigm [2], by basing the methodology on the xDAWN algorithm as defined by [3].

Farwell and Donchin P300 speller [2] presented alphanumeric characters and symbols to the subject; 36 in total; in a six by six grid as depicted in Figure 1(a) (the term symbol will refer to any alphanumeric character). This enabled subjects to select symbols sequentially and form words using brain waves and without requiring any movement. The methodology used derives from the oddball paradigm; first used in ERPs by Nancy, Kenneth and Steven [4]; where the subject is asked to distinguish between a common stimulus non-target and a rare stimulus target. In the case of the aforementioned P300 speller, 
the stimuli are visual, where each row and column of the spelling grid is augmented in a random order. The subject is asked to focus on the desired symbol 'target' and mentally count the number of times the row and column comprising the desired symbol is augmented. The action of counting heightens the ERP component. As a result of the target stimuli, an exogenous and spontaneous ERP potential known as P300; which is a positive deviation around 300ms after the stimuli; is evoked in the brain. The desired symbol is determined and predicted by the intersection of the target row and column. This prediction entails distinguishing between non-target i.e. rows/columns stimuli that does not generate a P300 component and target i.e. row/column stimuli that generate a P300 component.

In any recorded EEG signal, the P300 component which has a typical peak potential between 5-10 $\mathrm{V}$ [5], is embedded and masked by other brain activities (typical EEG signal $+-100 \mu \mathrm{V}$ ) such as muscular and/or ocular artefacts [1] leading to a very low Signal-to-Noise Ratio (SNR) of the P300 component. This indicates that it would be very difficult to detect the target stimuli from a single trial, which is denoted by a series of augmentation, in random order, of each of the six rows and six columns in our matrix (i.e. twelve augmentations per trial). A popular way to address the limited SNR of EEG is for each symbol to be spelled numerous consecutive times and the respective column/row epochs be averaged over a number of trials, thus cancelling components unrelated to stimulus onset [6]. A trade-off exists between increasing the number of trials per symbol (increases classification accuracy) and the number of symbols spelled per minute.

\subsection{The $x D A W N$ Spatial Filter}

The XDAWN process of spatial filtering is (1) a dimensionally reduction method that creates a subset of pseudo-channels (referred to as output channels) by a linear combination of the original channels and (2) it promotes the appealing part of the signal, such as ERPs, with respect to the noise. This is applied to the data before performing any classification such as LDA (Linear Discriminant Analysis) which was used in this paper. From an abstract point of view the xDAWN algorithm can be divided into (1) a least square estimate of the evoked responses, (2) a generalized Rayleigh quotient to estimate a set of spatial filters that maximize the SSNR (signal-to-signal-plus-noise-ratio).

The following is adapted from [3] and [7]. Let $\mathbf{X} \in \mathbb{R}^{\mathrm{S} \times \mathrm{C}}$ be the EEG data that contain ERPs and noise, with $S$ samples and $C$ channels. Let $\mathbf{A} \in \mathbb{R}^{\mathrm{ExC}}$ be the matrix of ERP signals, while $E$ is the number of temporal samples of the ERP (typically, $E$ is chosen to correspond to $600 \mathrm{~ms}$ or $1 \mathrm{~s}$ ). Let $\mathbf{N} \in \mathbb{R}^{\mathrm{SxC}}$ be the noise matrix which contains normally distributed noise. The ERPs position in the data is given by a Toeplitx matrix $\mathbf{D} \in \mathbb{R}^{\mathrm{ExS}}$. The data model is given by $\mathbf{X}=\mathbf{D}^{\mathbf{T}} \mathbf{A}+\mathbf{N}$. $\mathbf{A}$ is estimated by a least square estimate using a matrix inverse as shown in formula (1).

$$
\hat{\mathrm{A}}=\operatorname{argmin}=\|X-D A\|_{A}^{2}=\left(D^{T} D\right)^{-1} D^{T} X
$$

Let $\mathrm{W} \in \mathbb{R} S \times \mathrm{F}$ be the pseudo-channels while $\mathrm{F}$ represents the filters for projection. The result is the filtered data matrix $\tilde{X}=$ XW. According to (Rivet et al., 2009), the optimal filters W can be found by maximizing the SSNR as given by the generalized Rayleigh quotient in formula (2). The optimization problem is solved by combining a QRD (QR matrix decomposition) with an SVD (singular value decomposition). A more thorough explanation of the xDAWN algorithm can be found at [3].

$$
\hat{\mathrm{W}}=\arg \max _{W}=\frac{\operatorname{Tr}\left(W^{T} \hat{\mathrm{A}}^{T} D^{T} D \hat{\mathrm{A}} W\right)}{\operatorname{Tr}\left(W^{T} X^{T} X W\right)}
$$

\subsection{Subjects}

We enlisted five healthy subjects, three males and two females, aged 29-38 which voluntarily participated in this study. Four of the five subjects' native language was Maltese and the fifth subject's native language was English. All subjects spoke fluent English and were familiar with the symbols displayed on our screen. One of the subjects had previous experience using BCI and the P300 speller and will henceforth be referred as subject3 in the results. The other four subjects had never used or performed any BCI, nor have they ever seen a P300 speller. Three other subjects assisted in the initial experimentation where we assessed the viability of our equipment with the P300 component; however they did not take part in the official experiments and hence aren't included in the results. 


\subsection{Equipment Used}

The work reported herein is based on an OpenBCI 32-bit board (called Cyton) connected with an Electro-Cap using the international 10/20 system for scalp electrode placement in the context of EEG experiments as shown in Figure 1(b).

The Cyton board's microcontroller is the PIC32MX250F128B with a 32-bit processor and a maximum speed of 50MHz; storage of 32KB of memory and is Arduino compatible. The board uses the ADS1299 IC developed by Texas Instruments, which is an 8-Channel, 24-Bit, simultaneous sampling delta-sigma, Analogue-to-Digital Converter used for bio potential measurements. The system comes with a pre-programmed USB dongle for wireless communication which communicates with the low cost RFDuino RFD22301 microcontroller built on the OpenBCI board. An additional feature which is included in the OpenBCI board is a 3-axis accelerometer from ST with model LIS3DH. This can prove to be quite useful; such as, for sensing change in orientation of the head or sensing rough motion. A more thorough explanation of the hardware components of the Cyton can be found in our previous paper [1].

The Electro-Cap being used in our experiments has the fabric which is made from elastic spandex and has recessed pure tin wet electrodes directly attached to the fabric. The term wet electrodes type, implies that the use of an electrolyte gel is required to make effective contact with the scalp; otherwise it may result in impedance instability

\subsection{Experimental Procedure and Stimuli}

The EEG signals where sampled at $250 \mathrm{~Hz}$ (this being OpenBCIs default value) while the sampling precision was $24-$ bit. The recordings where stored anonymously as raw data in OpenVIBE's .ov format. These were later converted to a comma separated value (csv) files for offline analysis. Eight EEG electrodes where used in different regions of the scalp according to the International 10-20 System as shown in Figure 1(c). This system is the de facto standard for the placement of electrodes along the head. A more detailed explanation of the 10-20 System can be found in our previous paper [1]. The equipment we are using supports a maximum of sixteen electrodes. The Cyton board supports eight electrodes and an extension module (called Daisy) supports an additional eight electrodes. After initial analysis we did not see a major improvement between eight and sixteen electrodes and we have opted to exclude the use of the daisy module, hence the extra eight electrodes. A conventional lab study of EPs that includes a topographic representation may require additional electrodes; however in future studies, we are aiming to steer away from lab conditions.

The electrode positions $\mathrm{C} 3, \mathrm{Cz}, \mathrm{C} 4, \mathrm{P} 3, \mathrm{Pz}, \mathrm{P} 4, \mathrm{O} 1$ and $\mathrm{O} 2$ were selected. This is because the spatial amplitude dispersal of the P300 component is symmetric around $\mathrm{Cz}$ and its electrical potential is maximal in the midline region $(\mathrm{Cz}, \mathrm{Pz})$. It typically increases in magnitude from the frontal/occipital to parietal lobes [8]. The midline region is still widely used in almost all scientific papers related to P300 detection such as [9] and [10]. A referential montage was selected with the reference electrode being placed on the left earlobe A1 given that, in general, a mastoid or earlobe reference will produce a robust P300 response. The right ear lobe A2 is used as ground. The electrodes are referenced to electrode A1 as follows: Ch1: C3; Ch2: Cz; Ch3: C4; Ch4: P3; Ch5: Pz; Ch6: P4; Ch7: O1; Ch8: O2 as shown in Figure 1(c). Nonetheless and if required other types of montage can be reconstructed from the chosen montage by executing a simple mathematical operation (re-referencing) in the "offline" analysis, as explained in our previous paper [1].

In the induction session, each subject was briefed on the hardware being used and was shown a demonstration of an online P300 speller. Subsequently, the subjects' were informed on the following: (1) they would be performing the same experiment two consecutive times; in the training and online phase, both in lab conditions and (2) the symbols to spell were "P3SPELLER" respectively. Any subjects' query was answered at this stage.

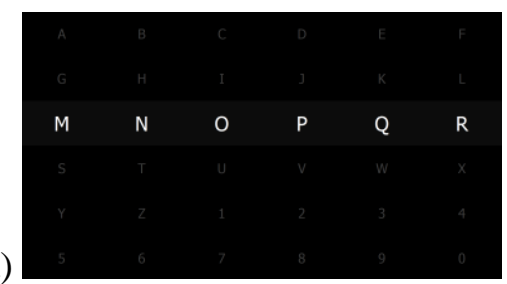

(b)

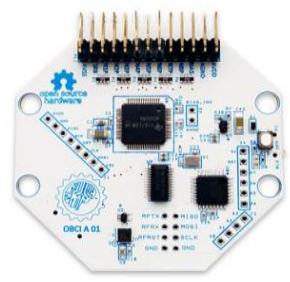

(c)

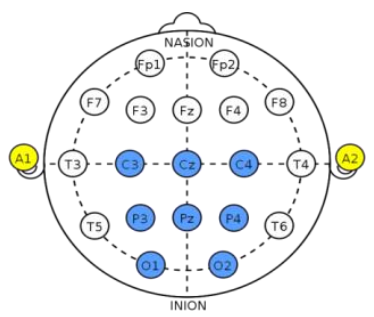

Fig. 1: (a) BCI "P300 Speller", (b) Cyton Board and Electro-CAP, (c) Electrode placement following the International 10-20

Before the start of the experiments, each subject was asked to relax for a few minutes in a seated position. The subject was seated approximately one meter away from the display. The researcher and his equipment were situated on the left side 
of the subject. The experiment was started when the subject was able to properly perform the task at hand and had no additional questions. Prior to the start of the experiment, the electrodes impedance was confirmed to be less than $5 \mathrm{~K} \Omega$.

The display presented to the subjects is shown in Figure 1(a) where 36 symbols were presented in a 6x6 matrix. The subjects' task was to visually focus their attention on the requested symbol, which was preceded by a cue i.e. one of the symbols was highlighted in blue at the beginning of the trials. The subject was asked to count the number of times the required symbol flashed which is then determined and predicted by the intersection of the (target) row and column. This prediction entails distinguishing between non-target i.e. rows/columns stimuli that does not generate a P300 component and target i.e. row/column stimuli that generate a P300 component. Each row and column in the matrix was augmented randomly for $100 \mathrm{~ms}$ and the delay between two successive augmentations was $80 \mathrm{~ms}$. This led to an interstimulus interval (ISI) of $180 \mathrm{~ms}$. For each symbol, six rows and six columns were augmented for fifteen repetitions and there was no inter-repetition delay. However there was a 3 s inter-trial period between the end of the trials of one symbol and the beginning of trials of the next symbol. This allowed the subject to focus the attention on the next symbol. At the end of each symbol run, the predicted symbol was highlighted in green which indicated whether the subject got the correct target symbol. The subjects were given a short break between experiments.

The training phase consisted of one session with 15 random symbols by fifteen trials each (i.e. 12 flashes of columns/rows per trial $* 15$ trials $=180$ flashes per symbol). In previous experiments with different subjects we have seen that there was no discernible difference in further increasing the number of trials per symbol or number of symbols in the training phase. According to previous success in the usability of the P300 speller with low cost equipment such as [10]; two criteria were established to evaluate the optimal number of symbols and trials in the training phase which correspond to two desired accuracies of $80 \%$ and $90 \%$ in an online system. The recording of the training phase took approximately 9 minutes. The online phase consisted of one session with the subjects spelling the symbols "P3SPELLER" consecutively. Similarly to the training phase, each symbol had 15 trials each. The recording of the online phase lasted approximately 6 minutes.

In total, there were 15 symbols spelled in the training phase and 9 symbols spelled in the online phase. Hence due to the matrix disposition there were in total 2700 flashes in the training phase, amongst which 450 were targets; and 1620 flashes in the online phase, amongst which 270 were targets. These values are per subject. The recordings where stored anonymously by referring to the subjects as subject1-subject5 respectively.

The online system was controlled by OpenViBE 2.0.0 which is a C++ based software platform designed for real-time processing of biosignal data. The acquisition server interfaces with the Cyton board and generates a standardized signal stream that is sent to the designer which in turn is used to construct and execute signal processing chains stored inside scenarios. The signal was obtained via the acquisition server which does not communicate directly with the Cyton board. Instead it provides a specific and dedicated set of drivers that does this task. The signal was obtained at a sampling rate of $250 \mathrm{~Hz}$ with $8 \mathrm{EEG}$ channels and 3 accelerometer (auxiliary) channels. The experiment paradigm was controlled by the OpenViBE designer where a number of scenarios in the "P300: Basic P300 Speller demo with XDAWN Spatial Filter" were executed in succession.

In the offline system, the captured raw data was converted from the proprietary OpenVIBE .ov extension to a more commonly used .csv format using a particular scenario aimed for this task. The outputs were two files in .csv format which contained the raw data and stimulations respectively. These files were later imported into MATLAB R2014a via the readtable command into MATLAB tables called samples and stims and were then converted to arrays via the table2array command. Subsequently any unnecessary rows and columns in the samples array were removed. These consisted of the first rows which contained the time header, channel names and sampling rate; the first column which contained the time(s) and the last three columns which stored the auxiliary data of the accelerometer. Next, we filtered out the stims array to include the target stimulations with code (33285); non-target stimulations (33286); visual stimulation stop (32780), which is the start of each flash of row or column; and segment start (32771), which is the start of each trial (12 flashes, 6 rows and 6 columns make up 1 trial). Additional data such as the sampleTime, samplingFreq and channelNames variables were extracted from the data and stored in the workspace. The samples array was later imported into EEGLAB for processing and for offline qualitative and quantitative analysis. The first process was to apply a band pass filter of $1-20 \mathrm{HZ}$ to eliminate the environmental electrical interference $(50 \mathrm{~Hz}$ or $60 \mathrm{~Hz}$ dependent on the country), to remove any signal harmonics and unnecessary frequencies which are not beneficial in our experiments and to remove the DC offset. Subsequently we import the event info (the stimulations - stim array) in EEGLAB with the format \{latency, type, duration\} in milliseconds. Next, the imported data was used in ERPLAB which is an add-on of EEGLAB, and is targeted for ERP analysis. Although the dataset in EEGLAB already contains information about all the individual events, we have created an eventlist structure in 
ERPLAB that consolidates this information and makes it easier to access and display; and also allows ERPLAB to add additional information which is not present in the original EEGLAB list of events. Subsequently we take every event we want to average together and assign that to a specific bin via the binlister. This must contain an abstract description of what kinds of event codes go into a particular bin. Subsequently we extracted the bin-based epochs via ERPLAB (not the EEGLAB version) and set the time period from $-0.2 \mathrm{~s}$ before the stimulus until $0.8 \mathrm{~s}$ after the stimulus. We have also used baseline correction (pre) since we wanted to subtract the average pre-stimulus voltage from each epoch of data. We have opted not to include any artefacts rejection, since this was not present in our online system. Lastly, we averaged our dataset ERPs to produce the required results which are shown in section 3.2.

\section{Results}

\subsection{Online Analysis}

Following the online experiments, we achieved the following results per subject, which are listed in Table 1. The letters to be spelled were P3SPELLER consecutively, while all percentages shown are rounded to the nearest one. The colour blue (bold in greyscale) denotes a bad prediction in the column. None of the subjects had an incorrect prediction on the row and/or in both the row and column. Subject1 and Subject 3 had an $89 \%$ success rate with Subject 1 having the letter $L$ predicted as letter $G$ i.e. the row prediction was correct but not the column, while Subject3 had the letter $R$ predicted as letter $F$ i.e. once again the row prediction was incorrect. All the other subjects (subject2, 4 and 5) had a 100\% success rate.

Table 1: Subject Results.

\begin{tabular}{|c|c|c|l|l|c|}
\hline Subject1 & Subject2 & Subject3 & Subject4 & Subject5 & Average \\
\hline $\begin{array}{c}\text { 8 out of 9 } \\
\text { P3SPEGLER } \\
\text { predicted }\end{array}$ & $\begin{array}{c}\text { P3SPELLER } \text { out } 9 \\
\text { predicted }\end{array}$ & $\begin{array}{c}\text { 8 out of 9 } \\
\text { P3SPLLEF } \\
\text { predicted }\end{array}$ & $\begin{array}{c}\text { 9 out of 9 } \\
\text { P3SPELLER } \\
\text { predicted }\end{array}$ & $\begin{array}{c}\text { 9 out of 9 } \\
\text { P3SPLLER } \\
\text { predicted }\end{array}$ & $95.6 \%$ \\
$89 \%$ success & $\begin{array}{l}100 \% \\
\text { success }\end{array}$ & $89 \%$ success & $\begin{array}{l}100 \% \\
\text { success }\end{array}$ & $\begin{array}{l}100 \% \\
\text { success }\end{array}$ & \\
\hline
\end{tabular}

\subsection{Offline Analysis}

The following figures represent the results that were processed in offline analysis. We have opted to present the averaged raw signals of the training and online session i.e. " 15 symbols for the training and 9 symbols for the online" with 15 trials per symbol; with 12 flashes of columns/rows per trial. The presented results are only passed through a band pass filter (1$20 \mathrm{~Hz}$ ) since this is needed to reduce the noise and unwanted frequencies, but it does not change the P300 signal i.e. it is essentially a pre-processing / conditioning step, it does not contribute directly to the analysis of the P300. In addition we have decided to refrain from using any artefact rejection in our offline analysis, since this was not present in our online system. A more through explanation of the processing can be found in Section 2.4. Furthermore we are not presenting the xDAWN spatial filters since our aim is to show the barest raw signal that is captured with our low fidelity equipment. Figure 2-6 represent Subject 1-5 results respectively while (a) represents the training session and (b) represents the online session. 

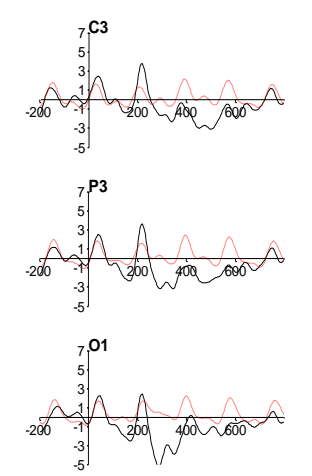

BIN1: Target

a)

BIN2: NonTarget

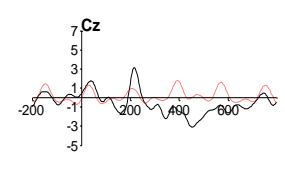

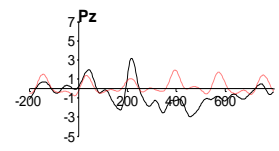

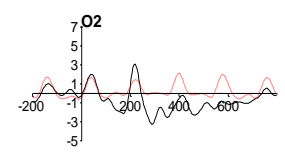

${ }_{7} \mathrm{C4}$
5.
3
1
-1
-3
-5

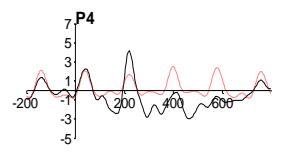

${ }_{7} \mathrm{Cz}$
5
3
1
-200
-3
-3.
-5

$\begin{array}{ccc}7_{7} \mathbf{P z} & \\ 5 \\ 3 \\ 1 \\ 1 \\ -200 \\ -1 . \\ -3 \\ -5\end{array}$

7.02
5
3
1
1
-1
-3
-5

BIN1: Target

b) $\begin{aligned} & 7 \\ & 7 \\ & 5 \\ & 3 \\ & 1 \\ &-200 \\ & 1 \\ &-3 \\ &-5\end{aligned} \quad 200 \quad 400 \quad 600$

$\left.\begin{array}{r}7_{-20} \mathbf{P 4} \\ 5 \\ 3 \\ 1 \\ -1 \\ -3 \\ -5\end{array}\right|_{200} A_{400} \bigwedge_{600}$

Fig. 2: Subject1's averaged ERP over all trials in (a) training session and (b) online session.
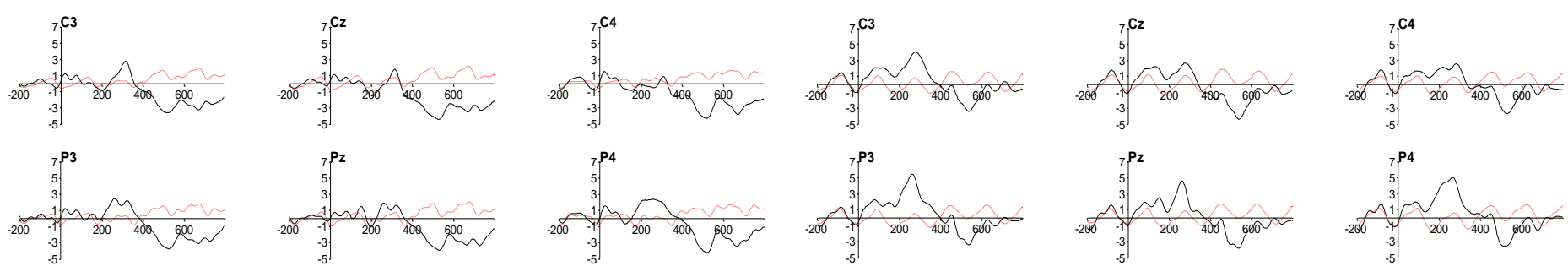

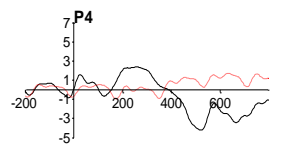
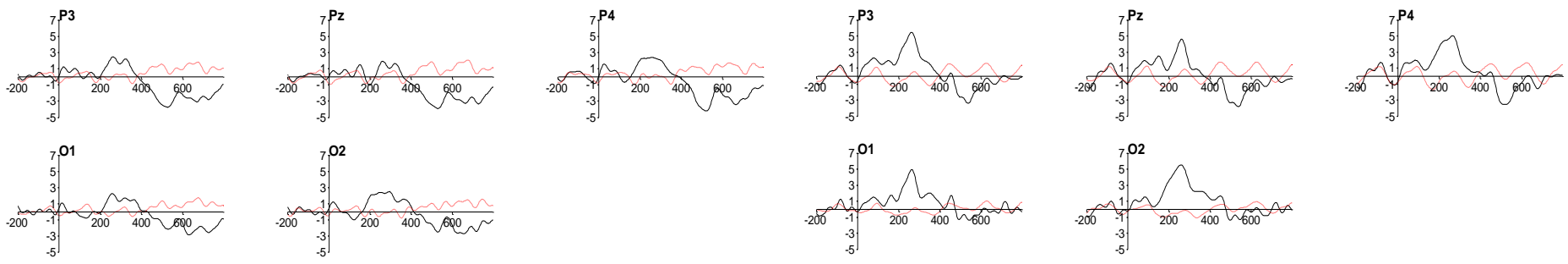

BIN1: Target

a)

BIN2: NonTarget

b)

BIN1: Target

Fig. 3: Subject2's averaged ERP over all trials in (a) training session and (b) online session.
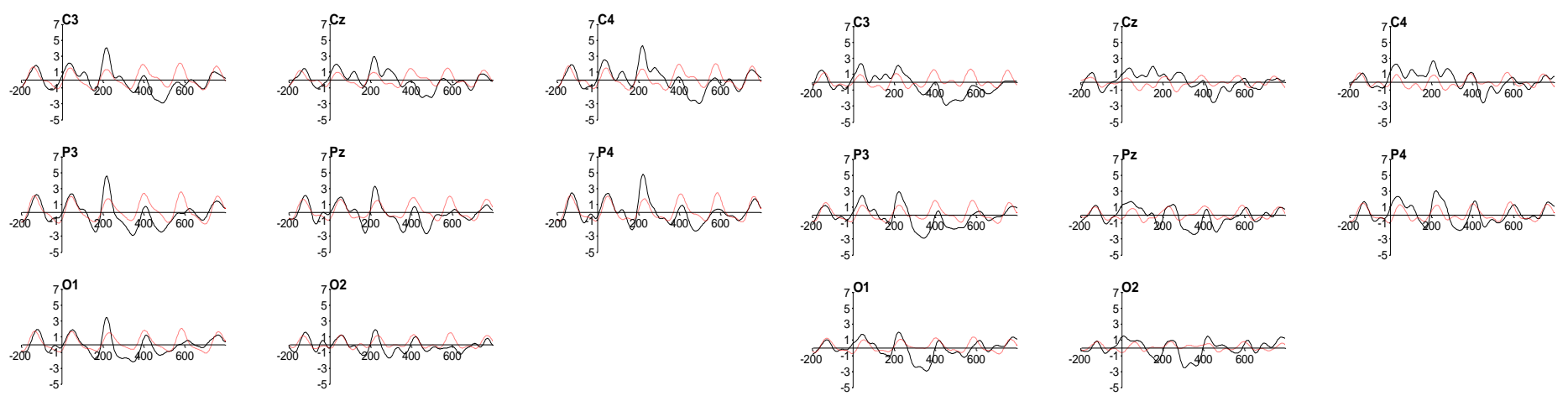

BIN1: Target

a)

b)

BIN2: NonTarget

Fig. 4: Subject3's averaged ERP over all trials in (a) training session and (b) online session. 

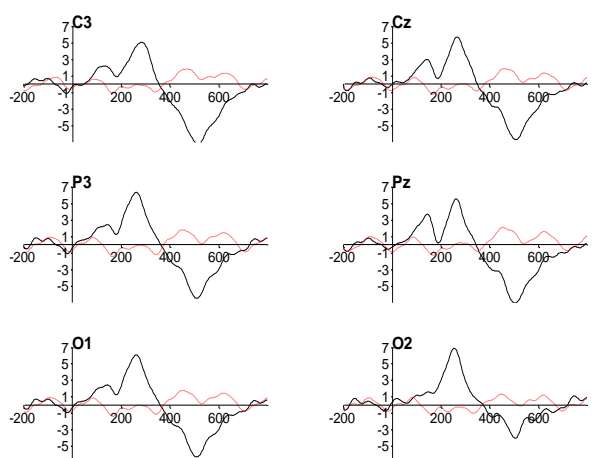

BIN1: Target

a)

BIN2: NonTarget

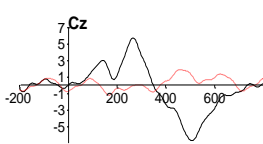

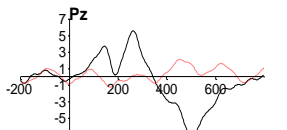
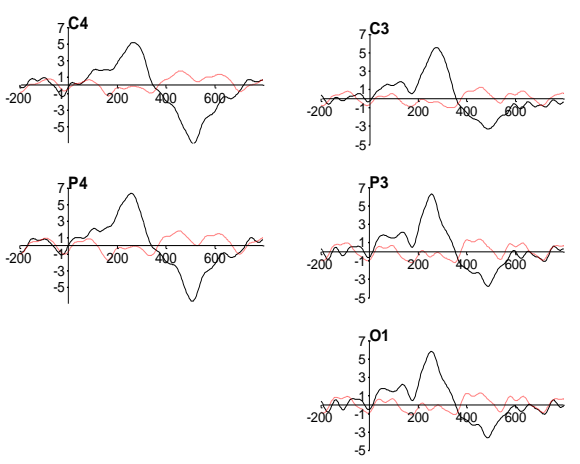

BIN1: Target b)
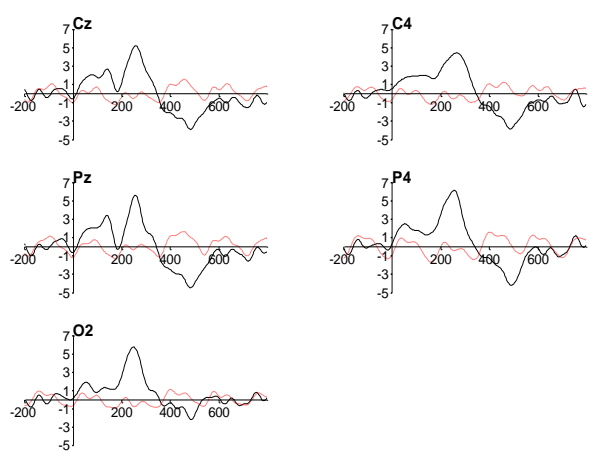

BIN2: NonTarget

Fig. 5: Subject4's averaged ERP over all trials in (a) training session and (b) online session.
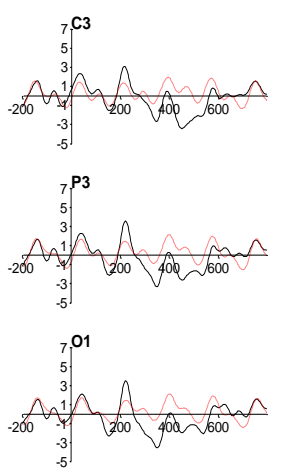

BIN1: Target
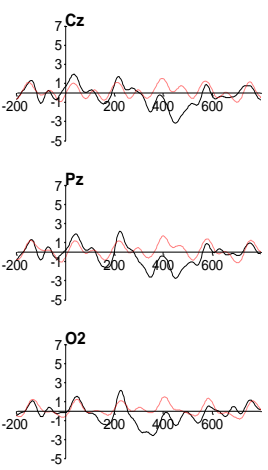

BIN2: NonTarget
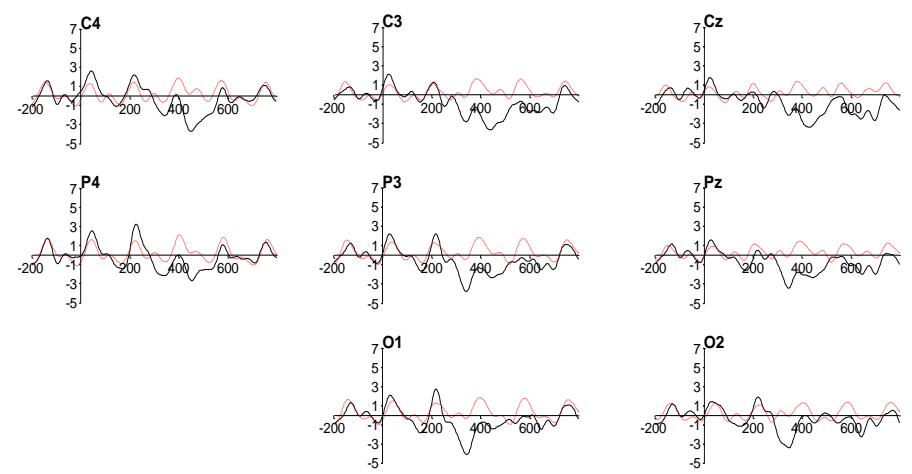

BIN1: Target

b)

BIN2: NonTarget

Fig. 6: Subject5's averaged ERP over all trials in (a) training session and (b) online session.
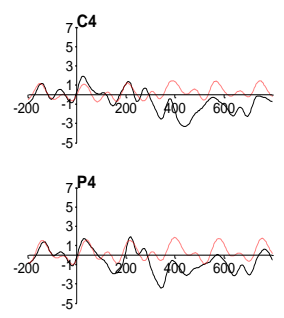

a)

\section{Conclusion}

The use of Brain Computer Interface (BCI) on the basis of Electroencephalography (EEG) signals has gained prominence over the past decade, especially with the institution of low cost devices, which made it accessible to a wide variety of researchers. However, due to the proliferation of these devices, including user-made equipment such as [11], an evident necessity to validate the equipment's suitability was present. Moreover in recent times, a number of researchers and end-users are using low fidelity equipment as a "black box" approach [12], without any qualitative testing on the equipment being used. The aim of this paper is to assess the functionality and consistency of our low cost hardware in the ability to successfully detect the P300 component in a reliable and consistent manner as a basis for future studies.

In continuation of our previous paper [1] we have resumed the validation of this equipment and we have improved performance upon [10] which was the last paper that utilized our equipment in conjunction with P300. In fact we have reduced the flashes per symbol from 24 down to 12 and have implemented the xDAWN algorithm which was not present in that study. Even though there are faster spellers, we have achieved the best published results using our specific equipment and the objective was not the speed of the application but the reliability of the equipment, with the aim to be used for further studies. Even though the success rate and speed might be related, we needed to establish a library of non-contaminated signals as a basis for comparisons in future work.

In this paper we have presented a practical approach in detecting the P300 component based on the xDAWN algorithm, while using low fidelity equipment. Five healthy subjects took part in our experiments where the promising results achieved show that we had an overall success rate of $95.6 \%$. This promising result allows us to proceed with our project, where we 
plan to introduce a number of distractions and assessing the ways and extents to which different degrees of distractions affect the detection success of the P300 component while using our low cost equipment.

\section{References}

[1] P. Schembri, R. Anthony, and M. Pelc, "Detection of Electroencephalography Artefacts using Low Fidelity Equipment," Proceedings of the 4th Int Conference on Physiological Computing Systems, pp. 65-75, 2017.

[2] L. A. Farwell and E. Donchin, "Talking off the top of your head: toward a mental prosthesis utilizing event-related brain potentials," Electroencephalography and cfinical Neurophysiology, vol. 70, pp. 510-523, 1988.

[3] B. Rivet, A. Souloumiac, V. Attina, and G. Gibert, "xDAWN Algorithm to Enhance Evoked Potentials: Application to BCI," IEEE Trasactions on Biomedical Eng., vol. 56, no. 8, pp. 2035-2043, 2009.

[4] N. Squires, K. Squires, and S. Hillyard, "Two varieties of long-latency positive waves evoked by unpredictable auditory stimuli in man," Electroencephalogr Clinical Neurophysiol, pp. 387-401, 1975.

[5] J. F. Peters and A. Skowron, eds., Transactions of Rough Sets V. Springer, 2006.

[6] B. Wittevrongel and M. M. Van Hulle, "Faster P300 Classifier Training Using Spatiotemporal Beamforming," International Journal of Neural Systems, vol. 26, no. 3, pp. 1650014-1:13, 2016.

[7] H. Woehrle et al., "An Adaptive Spatial Filter for User-Independent Single Trial Detection of Event-Related Potentials," IEEE Transactions On Biomedical Engineering, vol. 62, no. 7, pp. 1696-1705, 2015.

[8] R. Johnson, "On the neural generators of P300 component of ERP," Psychophysiology., vol. 30, pp. 90-97, 1993.

[9] D. De Venuto, V. F. Annese, and G. Mezzina, "An Embedded System Remotely Driving Mechanical Devices by P300 Brain Activity," IEEE Design, Automation and Test in Europe, pp. 1014-1019, 2017.

[10] J. Frey, "Comparison of an Open-hardware Electroencephalography Amplifier with Medical Grade Device in Braincomputer Interface Applications," in PhyCS, pp. 105-114, 2016.

[11] P. Wang, S. Li, M. Shao, and C. Liang, "A Low-Cost Portable Real-Time EEG Signal Acquisition System Based on DSP," Int Journal of Signal and Image Processing and Pattern Recognition, pp. 239-246, 2016.

[12] L. Lecoutre, S. Lini, C. Bey, Q. Lebour, and P. A. Favier, "Evaluating EEG Measures as a Workload Assessment in an Operational Video Game Setup," in PhyCS, pp. 112-117, 2015. 